NASZA DERMATOLOGIA Online OUR DERMATOLOGY Online

Source of Support:

FAPESP (Fundação de Apoio à

Pesquisa do Estado de São Paulo),

Process number: 2011/03230-1.

Competing Interests: None

\section{PEMPHIGUS AND PSYCHOLOGICAL STRESS: A REVIEW OF THE LITERATURE}

\author{
Aline Bicalho Matias, Ana Maria Ferreira Roselino
}

School of Medicine of Ribeirão Preto, University of São Paulo, São Paulo, Brazil

Corresponding author: M.Sc. Aline Bicalho Matias

alinebmatias@yahoo.com.br

\begin{abstract}
Introduction: Psychological stress has been associated with the course of several autoimmune skin diseases and reported a possible factor in triggering and aggravating for pemphigus in predisposed patients.

Aim: The aim of this study is to present an upgrade of the scientific literature on the relation between pemphigus and psychological stress.

Methods: To assure a comprehensive investigation, we have performed searches on LILACS, MedLine, PEPSIC, PubMed, SCOPUS and Web of Science databases. The terms used were pemphigus, psychological stress and psychological distress. We have selected works published on journals indexed in different online databases, without distinction as to language and date of the studies.

Results and Discussion: Initially, 22 works had fulfilled the selection criteria. After discarding publications which deviated from the subject, 9 works were selected for analysis. Among the selected articles, one was a theoretical review, five case studies, two case-control studies and one documental analysis. Publications discuss the importance of recognizing the influence of exogenous factors, such as psychological stress, on the development and evolution of pemphigus, since the health condition of the patients can be improved through the recognition, validation and treatment of their psychological issues, associating psychological assistance to the immunosuppressive treatment.

Conclusion: The report of stressor events by patients at the Dermatology Clinical is frequently observed, however, the relation between psychological stress and the development or aggravation of pemphigus is a recent subject among researchers of the field.
\end{abstract}

Key words: pemphigus foliaceus; pemphigus vulgaris; stress, psychological; review literature

\section{Introduction}

Pemphigus represents blistering autoimmune diseases, defined by IgG antibody deposits in the keratinocyte membranes of the epidermis, leading to acantholysis with consequent generation of blisters. While the morphology is well defined, its etiopathogenesis remains a subject of research [1].

Literature shows two mainly clinical forms of manifestations for pemphigus. Pemphigus foliaceus (PF), endemic in some countries, only affects the skin, and the autoantibodies primarily target the desmoglein 1, a cadherin-type protein constituent of the desmosomes, responsible for the adherence of keratinocytes $[2,3]$. Pemphigus vulgaris (PV) affects the skin and mucosae, and the autoantibodies linked to both desmoglein 1 and 3 , respectively. Desmoglein 3 prevails in the lower layers of epidermis and in the mucosae [4].

Brazilian and international medical-scientific production on pemphigus has increased significantly in recent years. Approximately 1.213 articles were published on the subject during the last 5 years; 258 in 2012 and 143 until august of 2013, according to the Virtual Health Library - BVS (http:// regional.bvsalud.org). The growth of the scientific knowledge has yielded visibility to the subject. On the other hand, the bigger exposure has revealed some gaps in the studies being performed. In spite of the growing number of publications, several aspects have been relatively under considered by science, especially regarding the influence of emotional aspects on the triggering and evolution of pemphigus.

A bibliographical search in BVS demonstrated the shortage of studies regarding the relation between psychological factors and pemphigus, especially in the case of stress, in spite of the psychological stress has been associated to the development of autoimmune diseases and can be one of the causes for its aggravation [5].

In 1965, Perry and Brunsting [6] reported that the symptoms of $\mathrm{PF}$ were aggravated due to emotional issues of the patient. On their turn, Brenner and Bar-Nathan [7] described two patients who developed PV under great emotional stress.

Considering those information, the aim of the present study is to present an upgrade of the scientific literature related to pemphigus, focusing on its relation to psychological stress, highlighting the profile of the works published in the most respectable sources in the field and discussing the trends indicated by such publications. 


\section{Methods}

In order to achieve the proposed objective, the literature review was made by combining terms related to pemphigus and psychological stress, as the scope of this study triggers works on the relation amongst stress and the development and evolution of pemphigus.

To assure a comprehensive search, we have used the LILACS, MedLine, PEPSIC, PubMed, SCOPUS and Web of Science databases. Such databases were chosen to give visibility to the scientific production of Brazilian and worldwide studies.

With the uniterm pemphigus we have localized 312 registered works in the LILACS, 6.899 in MedLine, 8.369 in PubMed, 10.191 in SCOPUS and 10.351 in Web of Science databases. In PEPSIC database were not found any work.

Given the objective of this review, the keywords psychological stress and psychological distress were added to pemphigus uniterm.

After the initial gathering of the publications indexed in these databases, their abstracts were read and analyzed according to inclusion/exclusion criteria. Selected works were fully accessed and analyzed in accordance with the article's scope. The analysis was made in order to retrieve the contributions provided by the selected articles.

As inclusion criterion, only works published in indexed journals from different online databases were selected, taking into consideration the fact that the systems available for bibliographical identification are very important resources for scientific disclosure, regardless the idiom, and without limitations as to the date of publication of the studies. As exclusion criterion, theses, dissertations, reviews, books and book chapters were excluded, as they do not undergo the evaluation process of peer review. Those publications which deviate from the studied subject were discarded.

\section{Result and Discussion}

Based on the search terms used, 22 works were compiled, until august 2013. In the MedLine and PubMed databases 15 works were found. In LILACS only one work was localized, which was discarded because it focused the coexistence of pemphigus and lichen in the oral mucosa. No works were found on the subject in the PEPSIC database. The 6 works found in Web of Science were also found in the other databases, except for one work, which was discarded because it focused quality of life. In the SCOPUS database we found 17 works, among which 12 were also found in PubMed and MedLine.

With the inclusion/exclusion criteria, the number of selected articles was reduced to 9 . The remaining works were discarded because their subjects deviated from what was proposed for this study, such as quality of life, environmental and occupational aspects, lichen, pediatric pemphigus, psoriasis, herpes, exposure to mercury, general skin diseases without mention to pemphigus, aggravation of the lesions caused by heat and pain syndrome.

Regarding the idiom, all the 9 selected publications are in English. As to the country where the study was held, we noticed the predominance of five countries: Israel (3), France (2), Italy (2), Croatia (1) and Egypt (1).

Concerning the specific characteristics of each study, we have found articles related to theoretical review [8], case reports [9$13]$, case-control studies $[14,15]$ and documental analysis [16]. The first study found during the previous decade discusses the case of a 68-year-old Ukrainian woman diagnosed with PV. Three months after her arrival in Israel, she began to present lesions on her skin and oral mucosa. The psychological evaluation discovered great sensibility and worry about her daughter and granddaughter, who had stayed in Ukraine. Even treated with drugs, lesions worsened for three weeks. With the beginning of treatment with psychiatric drugs, the patient presented an improvement of her mental health condition and became less anxious; then the clearance of the lesions occurred. This case report indicates the importance of recognizing the influence that psychological factors have on the PV pathogenesis among people with genetic predisposition to the condition [13].

Four years later, Cremniter et al. [9] pointed that, despite the genetic predisposition to pemphigus, the occurrence of cases in the same family is rare, suggesting that other aspects may be important in the disease's pathogenesis. The study deals with the investigation of stressful life events and personality disorders on 13 patients without previous treatment for pemphigus; 2 of those patients were diagnosed with PF and 11 with PV. Authors researched the impact of stressful life events which took place up to one year before the symptoms of pemphigus first appeared, the presence of personality disorders and symptoms of anxiety or depression. It was thus verified the presence of stressor events in 12 patients and some sort of personality disorder in 11 of them, what suggest that the presence of stressor events and personality disorders is probably not just a coincidence, as well as the potential development of pemphigus related to emotional stress.

Adding to the studies of Cremmiter et al, Morell-Dubois et al. [12], in an epidemiologic study with 10 participants, found evidence of the role of stress in the development of pemphigus, even when there is no personality disorder, and that stressful life events can aggravate or cause pemphigus.

The bibliographical review article [8], found in this search, discusses the data available in the literature from 1966 to 1998 about the role of stressful life events on the development or aggravation of skin conditions. In this review there is a paragraph related to pemphigus, in which the studies of Perry and Brunsting [6], Brenner and Bar-Nathan [7] and Cremniter et al [9] are presented and it is highlighted the lack of controlled studies as well as the small bibliographic production on the subject.

In another case report, published in 2004, it was presented a 58-year-old woman who lived under emotional stress since her childhood, in Poland, during the Holocaust. The first symptoms of PV appeared during the Gulf War, one month after the beginning of the treatment against tuberculosis with rifampicin. The authors of the study revealed that after the removal of the need for rifampicin by pulmonary lobotomy the pemphigus lesions reduced, except for the periods when the patient was under emotional stress. Such findings suggest the combination of factors in the development of the disease [10].

Šustić et al. [16] analyzed the files of patients from a hospital in Eastern Croatia in order to evaluate the epidemiology of blistering diseases through 10-year periods, from 1986 to 1990 and from 1990 to 1996, and to assess the effect of prolonged exposure to traumatic events during the Croatian War on the prevalence and incidence of the blistering diseases acquired.

In their findings, 45 patients developed some sort of blistering disease, 19 during the first analyzed period and 26 during the second, being PV the most common type. This indicates a larger incidence of blistering diseases during and immediately after the war. Therefore, prolonged psychological stress might be involved in the development of blistering diseases in the area studied. 
More recently, the study performed by Mazzotti et al. [11] presented the relation between psychological stress and dysfunctional investments on appearance, using interviews and self-applicable questionnaires about dysfunctional investments on appearance, and anxiety and nosocomial depression, among 78 patients with diagnosed PV and PF. Those patients who presented psychological stress also presented higher levels of dysfunctional investments on the appearance. However, the transversal nature of the study did not allow for the identification of the direction of such association, being impossible to affirm that a dysfunctional investment on the appearance causes psychological stress in patients of pemphigus or if the opposite occurs.

The bibliographical research resulted in a recent case-control Egyptian study. Ragab et al. [14] evaluated the serum TNFalpha levels in 10 patients with diagnosed PV, 4 with PF and 7 healthy individuals, correlating those levels with a history of stress. Patients of pemphigus were treated and observed for two months. The authors found higher serum TNF-alpha in PV patients compared to both PF patients and the control group. Four patients in the PV group presented aggravation of the condition and, among them, three suffered from high psychological stress one month before the development of the disease. It can be concluded that emotional stress is a factor which affects the prognostic of the disease, and it would be possible to suggest that the pre-treatment evaluation of serum levels of TNF-alpha in patients of pemphigus can be a guide to the prognostic and the selection of the appropriate treatment regimen.

The other case-control study [15] was performed with patients with diagnosed PV, and as control group were collected data of patients with diagnosed psoriasis. The study was not able to confirm a relation between the development or aggravation of PV and stress. However, the limitations of the study are widely discussed: its low number of samples, 17 patients, and the non-conformity of the questionnaire used for the evaluation of patients with different skin diseases.

\section{Conclusion}

The publications we have found focus mainly on reports of cases and the potential relation to previous experiences of stressor life events; only two studies used control groups [14,15]. Uncontrolled studies should be evaluated with precaution, as only controlled studies can produce strict enough evidence of the role of stressor events on the development or aggravation of skin diseases [8].

The selected articles deal with the importance of genetic predisposition to pemphigus. However, they point to the influence of exogenous factors on the pathogenesis of the disease, suggesting a combination of factors in the development of the disease.

The publications also discuss the importance of recognizing the influence of psychological stress on the development and evolution of pemphigus, since the health condition of the patients can be improved through the recognition, validation and treatment of their psychological issues, associating psychological assistance to the immunosuppressive treatment $[11,12,14]$.

The analysis of the literature review revealed that the relation between psychological stress and the development or aggravation of pemphigus is a recent subject among researchers of the field and the report of stressor events by patients at the Clinic of Dermatology is frequently observed. Although pemphigus is endemic in Brazil [2], with cases being reported since the beginning of the 20th century [17], we have not found Brazilian publications on the relation between pemphigus and psychological factors. It is therefore possible to observe a gap in the Brazilian scientific knowledge about the subject. And the need for more studies on the subject should be emphasized.

\section{REFERENCES}

1. Stanley JR, Amagai M: Pemphigus, bullous impetigo, and the Staphylococcal scalded-skin syndrome. N Engl J Med. 2006;355:1800-10.

2. Chiossi MP, Roselino AM: Endemic Pemphigus foliaceus („Fogo selvagem"): a series from the Northeastern region of the State of São Paulo, Brazil, 1973-1998. Rev Inst Med Trop São Paulo. 2001;43:5962.

3. Cianchini GMD, Corona R, Frezzolini A, Ruffeli M, Didoni B, Puddu P: Treatment of Severe Pemphigus With Rituximab. Arch Dermatol. 2007;143:1033-8.

4. Rocha-Alvarez R, Ortega-Loayza AG, Friedman H, Campbell I, Aoki V, Rivitti E A, et al: Endemic Pemphigus Vulgaris. Arch Dermatol. 2007;143:895-9.

5. Shoenfeld Y, Zandman-Goddard G, Stojanovich L, Cutolo M, Amital H, Levy Y, et al: The Mosaic of Autoimmunity: Hormonal and Environmental Factors Involved in Autoimmune Diseases. Isr Med Assoc J. 2008;10:8-12.

6. Perry HO, Brunsting LA: Pemphigus Foliaceus. Arch Dermatol. 1965;91:10-23.

7. Brenner S, Bar-Nathan EA: Pemphigus vulgaris triggered by emotional stress. J Am Acad Dermatol. 1984;11:524-5.

8. Picardi A, Abeni D: Stressful Life Events and Skin Diseases: Disentangling Evidence from Myth. Acta Psychother Psychosom. 2001;70:118-36.

9. Cremniter D, Baudin M, Roujeau JC, Prost C, Consoli SG, Francés $\mathrm{C}$ et al: Stressful Life Events as Potential Triggers of Pemphigus. Arch Dermatol. 1998;134:1486-7.

10. Goldberg I, Ingber A, Brenner S: Pemphigus vulgaris triggered by rifampin and emotional stress. Skinmed. 2004;3:294.

11. Mazzotti E, Mozzetta A, Antinone V, Alfani S, Cianchini G, Abeni D: Psychological distress and investment in one's appearance in patients with pemphigus. JEADV. 2011;25:285-9.

12. Morell-Dubois S, Carpentier O, Cottencin O, Queyrel V, Hachulla E, Hatron PY, et al.: Stressful Life Events and Pemphigus. Dermatology. 2008;16:104-8.

13. Tamir A, Ophir J, Brenner S: Pemphigus vulgaris triggered by emotional stress. Dermatology. 1994;189:210.

14. Ragab N, Abdallah M, El-Gohary E, Elewa R: Stress and serum TNF-alpha levels may predict disease outcome in patients with pemphigus: a preliminary study. Cutis. 2011;87:189-94.

15. Srebrnik A, Brenner S: Pemphigus vulgaris and Stress: A Psychological Evaluation of 17 Cases. Dermatol Psychosomat. 2001;2:191-4.

16. Šustić N, Ručević I, Barišić-Druško V: Epidemiology of Acquired Bullous Diseases in Eastern Croatia: A Retrospective Prewar to Postwar Study. Acta Dermatovenerol Croat. 2005;13:228-32.

17. Lindenberg A: Contribuição para o estudo da etiologia do pemphigus. Arq Dermatol Syphil. 1937;1:116-35.

Copyright by Aline Bicalho Matias, et al. This is an open access article distributed under the terms of the Creative Commons Attribution License, which permits unrestricted use, distribution, and reproduction in any medium, provided the original author and source are credited. 